\title{
Synthesis of Novel Pyridine-connected Piperidine and $2 H$-thiopyran Derivatives and their Larvicidal, Nematicidal, and Antimicrobial Activities
}

Anis Ahamed ${ }^{1}$, Ibrahim A. Arif ${ }^{1}$, Radhakrishnan Surendra Kumar ${ }^{2}$, Akbar Idhayadhulla $^{2 *}$, Selva raj Keerthana $^{2}$ and Aseer Manilal ${ }^{3}$

${ }^{1}$ Prince Sultan Research Chair for Environment and Wildlife, Department of Botany \& Microbiology, College of Sciences, King Saud University (KSU), Riyadh, Saudi Arabia.

$2^{*}$ Research Department of Chemistry, Nehru Memorial College (Affiliated to Bharathidasan University), Puthanampatti -621007, Tiruchirappalli District, Tamil Nadu, South India.

${ }^{3}$ Department of Medical Laboratory Sciences, College of Medicine and Health Sciences, Arba Minch University, Arba Minch, Ethiopia.

* Corresponding author: Akbar Idhayadhulla e-mail: a.idhayadhulla@gmail.com; idhayadhulla@nmc.ac.in, Phone: +919994265115

Received February $1^{\text {st }}, 2018$; Accepted July $26^{\text {th }}, 2018$.

DOI: $\underline{\text { http://dx.doi.org/10.29356/jmcs.v62i4.472 }}$

\begin{abstract}
A series of novel pyridine-connected piperidine derivatives (2a-g) and pyridine-connected $2 \mathrm{H}$ thiopyran derivatives (4a-g) were synthesized and evaluated for larvicidal, nematicidal, and antimicrobial activities. Compound $\mathbf{4 e}$ exhibited larvicidal activity against second instar larvae with an $\mathrm{LD}_{50}$ value of 0.8 $\mu \mathrm{g} / \mathrm{mL}$. In addition, $4 \mathbf{e}$ was most effective against root knot nematode Meloidogyne javanica, with an $\mathrm{LD}_{50}$ value of $3.2 \mu \mathrm{g} / \mathrm{mL}$. Compounds 2e (MIC: $4 \mu \mathrm{g} / \mathrm{mL}$ ) and 2d (MIC: $4 \mu \mathrm{g} / \mathrm{mL}$ ) exhibited high antibacterial activity against Klebsiella pneumonia, and Escherichia coli, respectively. Compounds 4b (MIC: $0.25 \mu \mathrm{g} / \mathrm{mL}$ ) and 4f (MIC: $2 \mu \mathrm{g} / \mathrm{mL}$ ) showed high antifungal activity against Candida albicans and Microsporum audouinii, respectively. Therefore, overall activity profiles envisages that compounds $\mathbf{2 e}, \mathbf{2 d}, \mathbf{4 e}, \mathbf{4 b}$, and $\mathbf{4 f}$ could be employed for the development of new classes of drugs with larvicidal, nematicidal, and antimicrobial activities.
\end{abstract}

Keywords: Larvicidal activity; Nematicidal activity; Antimicrobial activity; Piperidine; $2 H$-Thiopyran derivatives.

Resumen. Se sintetizaron una serie de nuevos derivados de piperidina conectados con piridina (2a-g) y derivados de $2 \mathrm{H}$-tiopirano conectados con piridina (4a-g) para evaluar las actividades larvicidas, nematicidas y antimicrobianas. El compuesto 4e exhibió actividad larvicida contra larvas de segundo estadio con un valor de $\mathrm{LD}_{50}$ de $0.8 \mu \mathrm{g} / \mathrm{mL}$. Además, $4 \mathrm{e}$ fue más efectivo contra el nematodo de nudo de la raíz Meloidogyne javanica, con un valor de $\mathrm{LD}_{50}$ de $3.2 \mu \mathrm{g} / \mathrm{mL}$. Los compuestos 2e (MIC: $4 \mu \mathrm{g} / \mathrm{ml}$ ) y 2d (MIC: $4 \mu \mathrm{g} / \mathrm{ml}$ ) mostraron una alta actividad antibacteriana contra la neumonía de Klebsiella y Escherichia coli, respectivamente. Los compuestos $\mathbf{4 b}$ (MIC: $0.25 \mu \mathrm{g} / \mathrm{ml}$ ) y $\mathbf{4 f}(\mathrm{MIC}: 2 \mu \mathrm{g} / \mathrm{ml}$ ) mostraron una alta actividad antifúngica contra Candida albicans y Microsporum audouinii, respectivamente. Por lo tanto, los perfiles de actividad general prevén que los compuestos $2 \mathbf{e}, \mathbf{2 d}, \mathbf{4 e}, \mathbf{4 b}$ y $\mathbf{4 f}$ podrían emplearse para el desarrollo de nuevas clases de fármacos con actividades larvicidas, nematicidas y antimicrobianas.

Palabras clave: Actividad larvicida; Actividad nematicida; Actividad antimicrobiana; Piperidina; Derivado 2H-Tiopirano. 


\section{Introduction}

Heterocyclic compounds, in particular piperidines, are considered biologically important and are used as vitamins, hormones, and antibiotics [1]. Piperidine nucleus is an important core of many drug molecules. Diverse pharmacological activities of piperidine and its analogs, including antihistamine, anticancer, and antibacterial properties, have been reported [2]. Pyrrolidine and piperidine occupy a unique place in the development of pharmacologically active substances by replacing the nucleus [3-4]. Piperidine derivatives have been reported to possess significant pharmacological activities such as larvicidal [5], antiinflammatory [6], local anesthetic [7], anticancer [8], and antimicrobial properties [9].

Thiopyran structures are considered as one of the most important classes of sulfur-containing heterocycles because of their usefulness in accessing certain natural and unnatural products. There has been an increased focus on sulfur-containing heterocyclic compounds because a broad range of biological activities related to the structure have been reported [10]. Thiopyran compounds shows antibacterial [11], antihyperplasia [12], anti-psychotic [13], analgesic, and anti-inflammatory [14] activities.

Mosquito larvae can be controlled by insecticides [15-17] and the best larvicides are natural products and heterocyclic compounds. For instance, $N^{\prime}$-tert-butyl- $N, N^{\prime}$-dibenzoylhydrazine (RH-5849) was reported as the first nonsteroidal ecdysone agonist in the mid-1980s [18]. The mosquito borne diseases not only cause high levels of morbidity and mortality but also cause great economic loss to health care. Recent estimates from the World Health Organization (WHO) evidenced that malaria accounts for at least 500 million infections and 3 million deaths annually. The prevalence of dengue fever has increased over the last 50 years, and over 2 billion people are under risk in more than 100 countries [19]. In the wake of this, there is an urgent need to develop new insecticides that are environmentally-friendly and biodegradable and can target specific mosquitoes.

Thousands of crops and trees are susceptible, and the disease caused by phytonematodes results in huge agricultural loss annually [20]. Levamisole is used to treat parasitic worm infections [21]. Plants infested by nematodes show retarded growth and development, as well as loss in the quality and quantity of the harvest. Due to environmental problems, nematicides, such as dibromochloropropane (DBCP) and ethylenedibromide (EDB) were withdrawn from the market. Howbeit, some simple coumarins, furocoumarins, and dicoumarolums, display excellent nematicidal activity, and their skeletons have drawn interest for the development of efficient nematicides [15].

Considering these observations, in the present study, we synthesized a series of pyridine-connected piperidine derivatives (2a-g) and $2 H$-thiopyran (4a-g) derivatives, and screened them for larvicidal, nematicidal, and antimicrobial activities.

\section{Experimental}

\section{Materials}

All chemicals were purchased from Merck, Sigma-Aldrich and used without further purification. The solvents were dried and distilled prior to use. Merck pre-coated silica gel plates with a fluorescent indicator were used for analytical thin-layer chromatography (TLC). Flash column chromatography was performed using silica gel (Merck). EtOAc-hexane was used as the eluting solvent for TLC and column chromatography. Melting points were recorded in open capillary tubes and were uncorrected. The IR spectra $(\mathrm{KBr})$ were recorded in $\mathrm{KBr}$ on a Shimadzu $8201 \mathrm{pc}\left(4000-400 \mathrm{~cm}^{-1}\right)$. The ${ }^{1} \mathrm{H}$ NMR and ${ }^{13} \mathrm{C}$ NMR spectra were recorded on a Bruker DRX-300 MHz. The elemental analysis $(\mathrm{C}, \mathrm{H}$, and $\mathrm{N})$ was conducted using an Elementer analyzer model (Varian EL III). The purity of the compounds was checked by TLC with silica gel plates.

\section{General procedure for synthesis of 2-(hydrazonomethyl)pyridine (HMP)}

A mixture of pyridine 2 -aldehyde $(0.1 \mathrm{~mol})$ and hydrazine hydrate $(0.1 \mathrm{~mol})$ in ethanol was heated at refluxed for $5 \mathrm{~min}$. After completed reaction the solid material was filtered and washed with distilled water. The product was confirmed by TLC (hexane-EtOAc, 4:1, v/v). The product (HMP) was purified by flash column chromatography. 


\section{General procedure for synthesis of (E)-2-(((2,6-diphenylpiperidin-4-ylidene)hydrazono) methyl)pyridine (2a-g)}

A mixture of compound 1a $(0.1 \mathrm{~mol})$ and 2-(hydrazonomethyl)pyridine $(0.1 \mathrm{~mol})$ in ethanol was heated at refluxed for $2 \mathrm{~h}$. The product $\mathbf{2 a}$ was purified by flash column chromatography on silica gel using hexane/EtOAc.

Yellow solid: yield $81 \%$. mp $129-131{ }^{\circ} \mathrm{C}$; IR $\left(\mathrm{KBr}, \mathrm{cm}^{-1}\right)$ : $3045,3010,1671,802,712 ;{ }^{1} \mathrm{H}$ NMR $(300$ MHz, DMSO-d6): $\delta 11.15(1 \mathrm{H}, \mathrm{s}, \mathrm{NH}), 8.61(1 \mathrm{H}, \mathrm{d}, J=7.4 \mathrm{~Hz}$, pyridine $), 7.82(1 \mathrm{H}, \mathrm{d}, J=7.2 \mathrm{~Hz}$, pyridine), $7.71(1 \mathrm{H}, \mathrm{dd}, J=7.1, J=7.0 \mathrm{~Hz}$, pyridine), $7.61(1 \mathrm{H}, \mathrm{dd}, J=7.1 \mathrm{~Hz}, J=7.4 \mathrm{~Hz}$, pyridine), $7.59-7.51(10 \mathrm{H}, \mathrm{m}$, aryl), $7.48(1 \mathrm{H}, \mathrm{s},-\mathrm{HC}=\mathrm{N}-), 3.72(2 \mathrm{H}, \mathrm{dd}, J=13.7 \mathrm{~Hz}, J=13.10 \mathrm{~Hz}, 2 \mathrm{C}-\mathrm{H}, 6 \mathrm{C}-\mathrm{H}), 2.51(2 \mathrm{H}, \mathrm{dd}, J=13.6 \mathrm{~Hz}$, $J=13.04 \mathrm{~Hz}, 3 \mathrm{C}-\mathrm{Heq}, 5 \mathrm{C}-\mathrm{Heq}), 1.34(2 \mathrm{H}, \mathrm{dd}, J=13.4 \mathrm{~Hz}, J=13.08 \mathrm{~Hz}, 3 \mathrm{C}-\mathrm{Hax}, 5 \mathrm{C}-\mathrm{Hax}) ;{ }^{13} \mathrm{C}$ NMR $\left(75 \mathrm{MHz}, \mathrm{DMSO}-d_{6}\right) \delta: 168.3(1 \mathrm{C}, \mathrm{C}=\mathrm{N}), 164.2(1 \mathrm{C}, \mathrm{C}=\mathrm{N}), 153.9(1 \mathrm{C}), 146.7(1 \mathrm{C}), 136.9(1 \mathrm{C}), 128.8-127.1$ (12C, aryl), 126.1 (1C), 121.0 (1C), 46.8 (2C), 46.1 (1C), 34.5 (1C); EI MS $m / z$ (rel.int): $354[\mathrm{M}]^{+}$(26), 277 (13), 264 (24), 244 (24), 237 (100), 161 (11), 85 (10), 73 (21), 44 (18); Anal C 77.91\%, H 6.21\%, N 15.80\%, Calcd for $\mathrm{C}_{23} \mathrm{H}_{22} \mathrm{~N}_{4}, \mathrm{C} 77.94 \%$, H $6.26 \%$, N $15.81 \%$.

(E)-2-((2,6-Bis(4-chlorophenyl)piperidin-4-ylidene)hydrazono)methyl)pyridine (2b). Yellow solid: yield 85\%; mp 137-139 ${ }^{\circ} \mathrm{C}$; IR( $\left.\mathrm{KBr}, \mathrm{cm}^{-1}\right): 3082,3020,1681,862,831,710 ;{ }^{1} \mathrm{H}$ NMR (DMSO-d 6 ): $\delta$ $11.23(1 \mathrm{H}, \mathrm{s}, \mathrm{NH}), 8.61(1 \mathrm{H}, \mathrm{d}, J=7.4 \mathrm{~Hz}$, pyridine $), 7.82(1 \mathrm{H}, \mathrm{d}, J=7.2 \mathrm{~Hz}$, pyridine $), 7.71(1 \mathrm{H}, \mathrm{dd}, J=7.1$, $J=7.0 \mathrm{~Hz}$, pyridine $), 7.61(1 \mathrm{H}, \mathrm{dd}, J=7.1 \mathrm{~Hz}, J=7.4 \mathrm{~Hz}$, pyridine $), 7.50(1 \mathrm{H}, \mathrm{s},-\mathrm{HC}=\mathrm{N}-) 7.47(4 \mathrm{H}, \mathrm{d}, J=$ $7.2 \mathrm{~Hz}$, aryl $), 7.43-7.39(2 \mathrm{H}, \mathrm{d}, J=4.8 \mathrm{~Hz}$, aryl $), 7.48-7.44(2 \mathrm{H}, \mathrm{d}, J=4.8 \mathrm{~Hz}$, aryl $), 3.79(2 \mathrm{H}, \mathrm{dd}, J=13.7 \mathrm{~Hz}$, $J=11.34 \mathrm{~Hz}, 2 \mathrm{C}-\mathrm{H}, 6 \mathrm{C}-\mathrm{H}), 3.57(2 \mathrm{H}, \mathrm{dd}, J=13.4 \mathrm{~Hz}, J=11.34 \mathrm{~Hz}, 3 \mathrm{C}-\mathrm{Heq}, 5 \mathrm{C}-\mathrm{Heq}), 2.89$ (2H, dd, $J=$ $11.46 \mathrm{~Hz}, J=11.46 \mathrm{~Hz}, 3 \mathrm{C}-\mathrm{Hax}, 5 \mathrm{C}-\mathrm{Hax}) ;{ }^{13} \mathrm{C}$ NMR(DMSO-d 6 ): $\delta 166.1(1 \mathrm{C}, \mathrm{C}=\mathrm{N}), 156.2(1 \mathrm{C}, \mathrm{C}=\mathrm{N}), 152.4$ (1C), 146.4 (1C), 136.5 (1C), 126.3 (1C), 121.3 (1C), 128.9 (4C), 128.0 (4C), 140.5 (2C), 131.8 (2C, $\underline{\mathrm{C}}-\mathrm{Cl})$, 61.8 (1C), 54.5 (1C), 46.1 (1C), 36.8 (1C); EIMS m/z (rel.int): $423[\mathrm{M}]^{+}$(26), 346 (14), 333 (18), 318 (23), 306 (32), 237 (100), 161 (16), 85 (28), 73 (19), 44 (10); Anal C 65.46\%, H 4.72\%, N 13.10\%, Calcd for $\mathrm{C}_{23} \mathrm{H}_{20} \mathrm{Cl}_{2} \mathrm{~N}_{4}, \mathrm{C} 65.25 \%, \mathrm{H} 4.76 \%$, N $13.01 \%$.

(E)-4,4'-(4-((Pyridin-2-ylmethylene)hydrazono)piperidine-2,6-diyl)diphenol (2c). Yellow solid: yield $80 \%$; mp $145-149^{\circ} \mathrm{C}$; IR $\left(\mathrm{KBr}, \mathrm{cm}^{-1}\right): 3046,1640,1451,828,711 ;{ }^{1} \mathrm{H}$ NMR (DMSO-d 6 ): $\delta 11.71(1 \mathrm{H}, \mathrm{s}$, $\mathrm{NH}), 9.56(2 \mathrm{H}, \mathrm{s}, \mathrm{OH}), 8.61(1 \mathrm{H}, \mathrm{d}, J=7.4 \mathrm{~Hz}$, pyridine $), 7.82(1 \mathrm{H}, \mathrm{d}, J=7.2 \mathrm{~Hz}$, pyridine $), 7.71(1 \mathrm{H}, \mathrm{dd}, J=$ $7.1 \mathrm{~Hz}, J=7.0 \mathrm{~Hz}$, pyridine), $7.61(1 \mathrm{H}, \mathrm{dd}, J=7.1 \mathrm{~Hz}, J=7.4 \mathrm{~Hz}$, pyridine), 7.19-7.10 (4H, d, $J=8.2 \mathrm{~Hz}$, aryl), 6.70-6.67 $(4 \mathrm{H}, \mathrm{d}, J=7.80 \mathrm{~Hz}$, aryl), $7.56(1 \mathrm{H}, \mathrm{s},-\mathrm{HC}=\mathrm{N}-), 3.74(2 \mathrm{H}, \mathrm{dd}, J=13.74 \mathrm{~Hz}, J=13.10 \mathrm{~Hz}$, $2 \mathrm{C}-\mathrm{H}, 6 \mathrm{C}-\mathrm{H}), 3.32(2 \mathrm{H}, \mathrm{dd}, J=13.62 \mathrm{~Hz}, J=11.67 \mathrm{~Hz}, 3 \mathrm{C}-\mathrm{Heq}, 5 \mathrm{C}-\mathrm{Heq}), 2.24(2 \mathrm{H}, \mathrm{dd}, J=13.68 \mathrm{~Hz}, J=$ $11.39 \mathrm{~Hz}, 3 \mathrm{C}-\mathrm{Hax}, 5 \mathrm{C}-\mathrm{Hax}) ;{ }^{13} \mathrm{C}$ NMR (DMSO-d 6 ): $\delta 168.7(1 \mathrm{C}, \mathrm{C}=\mathrm{N}), 159.2(2 \mathrm{C}, \underline{\mathrm{C}-\mathrm{OH}}), 156.4(1 \mathrm{C}, \mathrm{C}=\mathrm{N})$, 153.2 (1C), 147.0 (1C), 136.1 (1C), 135.9 (2C), 128.3 (4C), 126.4 (1C), 121.3 (1C), 115.6 (4C), 62.6 (1C), 55.8 (1C), 46.1 (1C), 36.8 (1C); EIMS m/z (rel.int): $386[\mathrm{M}]^{+}$(21), 309 (18), 231 (27), 269 (17), 237 (100), 161 (21), 85 (17), 73 (20), 44 (11); Anal C 71.46\%, H 5.77\%, N 14.55\%, Calcd for $\mathrm{C}_{23} \mathrm{H}_{22} \mathrm{~N}_{4} \mathrm{O}_{2}, \mathrm{C} 71.48 \%, \mathrm{H}$ $5.74 \%, \mathrm{~N} 14.50 \%$.

(E)-2-((2,6-Bis(4-nitrophenyl)piperidin-4-ylidene)hydrazono)methyl)pyridine (2d). Yellow solid: yield $74 \%$; mp 155-159 ${ }^{\circ} \mathrm{C}$; IR $\left(\mathrm{KBr}, \mathrm{cm}^{-1}\right): 3079,3021,1684,1531,808,721 ;{ }^{1} \mathrm{H}$ NMR (DMSO-d 6 ): $\delta$ $11.28(1 \mathrm{H}, \mathrm{s}, \mathrm{NH}), 8.61(1 \mathrm{H}, \mathrm{d}, J=7.4 \mathrm{~Hz}$, pyridine $), 8.45(4 \mathrm{H}, \mathrm{d}, J=7.2 \mathrm{~Hz}$, aryl $), 7.82(1 \mathrm{H}, \mathrm{d}, J=7.2 \mathrm{~Hz}$, pyridine), $7.72(1 \mathrm{H}, \mathrm{s},-\mathrm{HC}=\mathrm{N}-), 7.71(1 \mathrm{H}, \mathrm{dd}, J=7.1 \mathrm{~Hz}, J=7.0 \mathrm{~Hz}$, pyridine $), 7.61(1 \mathrm{H}, \mathrm{dd}, J=7.1 \mathrm{~Hz}, J=$ $7.4 \mathrm{~Hz}$, pyridine), $7.29(4 \mathrm{H}, \mathrm{d}, J=7.2 \mathrm{~Hz}$, aryl ), $3.79(2 \mathrm{H}$, dd, $J=13.7 \mathrm{~Hz}, J=13.8 \mathrm{~Hz}, 2 \mathrm{C}-\mathrm{H}, 6 \mathrm{C}-\mathrm{H}), 3.35$ $(2 \mathrm{H}, \mathrm{dd}, J=13.8 \mathrm{~Hz}, J=11.6 \mathrm{~Hz}, 3 \mathrm{C}-\mathrm{Heq}, 5 \mathrm{C}-\mathrm{Heq}), 2.11(2 \mathrm{H}, \mathrm{d}, J=11.39 \mathrm{~Hz}, 3 \mathrm{C}-\mathrm{Hax}, 5 \mathrm{C}-\mathrm{Hax}) ;{ }^{13} \mathrm{C}$ NMR (DMSO-d $\left.{ }_{6}\right): \delta 167.1(1 \mathrm{C}, \mathrm{C}=\mathrm{N}), 157.7(1 \mathrm{C}, \mathrm{C}=\mathrm{N}), 153.7(1 \mathrm{C}), 148.4(2 \mathrm{C}), 146.4(1 \mathrm{C}), 145.2\left(2 \mathrm{C}, \underline{\mathrm{C}}-\mathrm{NO}_{2}\right)$, 136.1 (1C), $126.7(1 \mathrm{C}), 125.9$ (4C), 124.8 (4C), 121.5 (1C), 61.8 (1C), 54.5 (1C), 46.1 (1C), 36.8 (1C); EIMS $\mathrm{m} / z$ (rel. int): $444[\mathrm{M}]^{+}$(26), 367 (16), 354 (10), 339 (28), 327 (32), 237 (100), 161 (27), 85 (19), 73 (15), 44 (5); Anal C 62.16\%, H 4.57\%, N 18.92\%, Calcd for $\mathrm{C}_{23} \mathrm{H}_{20} \mathrm{~N}_{6} \mathrm{O}_{4}, \mathrm{C} 62.16 \%, \mathrm{H}, 4.54 \%, \mathrm{~N} 18.91 \%$.

(E)-2-((2,6-Bis(4-methoxyphenyl)piperidin-4-ylidene)hydrazono)methyl)pyridine (2e). Yellow solid: yield $76 \%$; mp 154-158 ${ }^{\circ} \mathrm{C}$; IR $\left(\mathrm{KBr}, \mathrm{cm}^{-1}\right)$ : 3085, 3023, 1671, 806, 729; ${ }^{1} \mathrm{H}$ NMR(DMSO-d 6 ): $\delta 11.23$ $(1 \mathrm{H}, \mathrm{s}, \mathrm{NH}), 8.61(1 \mathrm{H}, \mathrm{d}, J=7.4 \mathrm{~Hz}$, pyridine), $7.90(1 \mathrm{H}, \mathrm{s},-\mathrm{HC}=\mathrm{N}-), 7.82(1 \mathrm{H}, \mathrm{d}, J=7.2 \mathrm{~Hz}$, pyridine), 7.71 
(1H, dd, $J=7.1 \mathrm{~Hz}, \mathrm{~J}=7.0 \mathrm{~Hz}$, pyridine), $7.61(1 \mathrm{H}, \mathrm{dd}, J=7.1 \mathrm{~Hz}, J=7.4 \mathrm{~Hz}$, pyridine), 7.22-7.20 (4H, d, $J$ $=7.2 \mathrm{~Hz}, \operatorname{aryl}), 6.35-6.31(4 \mathrm{H}, \mathrm{d}, J=7.2 \mathrm{~Hz}, \operatorname{aryl}), 3.85\left(6 \mathrm{H}, \mathrm{s},-\mathrm{OCH}_{3}\right), 3.72(2 \mathrm{H}, \mathrm{dd}, J=13.7 \mathrm{~Hz}, J=11.6$ $\mathrm{Hz}, 2 \mathrm{C}-\mathrm{H}, 6 \mathrm{C}-\mathrm{H}), 3.49(2 \mathrm{H}, \mathrm{dd}, J=13.4 \mathrm{~Hz}, J=11.6 \mathrm{~Hz}, 3 \mathrm{C}-\mathrm{Heq}, 5 \mathrm{C}-\mathrm{Heq}), 2.17(2 \mathrm{H}, \mathrm{dd}, J=13.3 \mathrm{~Hz}, J=$ $11.4 \mathrm{~Hz} 3 \mathrm{C}-\mathrm{Hax}, 5 \mathrm{C}-\mathrm{Hax}) ;{ }^{13} \mathrm{C}-\mathrm{NMR}\left(\mathrm{DMSO}_{\mathrm{d}}\right): \delta 168.2(1 \mathrm{C}, \mathrm{C}=\mathrm{N}), 158.3(1 \mathrm{C}, \mathrm{C}=\mathrm{N}), 157.9(2 \mathrm{C}, \mathrm{C}-$ $\left.\mathrm{OCH}_{3}\right), 153.3(1 \mathrm{C}), 147.2(1 \mathrm{C}), 137.8$ (1C), $126.2(1 \mathrm{C}), 121.5$ (1C), $114.8(4 \mathrm{C}), 126.9(4 \mathrm{C}), 134.7(2 \mathrm{C}), 61.8$ (1C), $55.1\left(2 \mathrm{C}, \mathrm{OCH}_{3}\right), 54.5$ (1C), 46.1 (1C), 36.8 (1C); EIMS $m / z$ (rel.int): 414 [M] $]^{+}$(26), 336 (21), 310 (19), 297 (34), 237 (100), 161 (10), 85 (10), 73 (18), 44 (6); Anal C 72.46\%, H 6.07\%, N 13.95\%, Calcd for $\mathrm{C}_{25} \mathrm{H}_{26} \mathrm{~N}_{4} \mathrm{O}_{2} \mathrm{C} 72.44 \%$, H 6.32\%, N $13.52 \%$.

(E)-2-(((2,6-Di-p-tolylpiperidin-4-ylidene)hydrazono)methyl)pyridine (2f). Yellow solid; yield $81 \%$; mp 167-171 ${ }^{\circ} \mathrm{C}$; IR (KBr, cm $\left.{ }^{-1}\right): 3094,3013,1651,825,710 ;{ }^{1} \mathrm{H}$ NMR (DMSO-d 6 ): $\delta 11.26(1 \mathrm{H}, \mathrm{s}, \mathrm{NH})$, $8.61(1 \mathrm{H}, \mathrm{d}, J=7.4 \mathrm{~Hz}$, pyridine), $7.93(1 \mathrm{H}, \mathrm{s}, \mathrm{HC}=\mathrm{N}-), 7.82(1 \mathrm{H}, \mathrm{d}, J=7.2 \mathrm{~Hz}$, pyridine $), 7.71(1 \mathrm{H}, \mathrm{dd}, J=$ $7.1 \mathrm{~Hz}, J=7.0 \mathrm{~Hz}$, pyridine), $7.61(1 \mathrm{H}, \mathrm{dd}, J=7.1 \mathrm{~Hz}, J=7.4 \mathrm{~Hz}$, pyridine), $7.45-7.40(4 \mathrm{H}, \mathrm{d}, J=7.2 \mathrm{~Hz}$, aryl), 7.29-7.26 (4H, d, $J=7.2 \mathrm{~Hz}$, aryl), $3.72(2 \mathrm{H}, \mathrm{dd}, J=13.7 \mathrm{~Hz}, J=11.6 \mathrm{~Hz}, 2 \mathrm{C}-\mathrm{H}, 6 \mathrm{C}-\mathrm{H}), 3.36(2 \mathrm{H}$, dd, $J=13.5 \mathrm{~Hz}, J=11.6 \mathrm{~Hz}, 3 \mathrm{C}-\mathrm{Heq}, 5 \mathrm{C}-\mathrm{Heq}), 2.28\left(6 \mathrm{H}, \mathrm{s}, \mathrm{CH}_{3}\right), 2.08(2 \mathrm{H}, \mathrm{dd}, J=13.3 \mathrm{~Hz}, J=11.36 \mathrm{~Hz}, 3 \mathrm{C}-$ Hax, 5C-Hax); ${ }^{13} \mathrm{C}-\mathrm{NMR}\left(\mathrm{DMSO}_{6}\right): \delta 167.1(1 \mathrm{C}, \mathrm{C}=\mathrm{N}), 157.6(1 \mathrm{C}, \mathrm{C}=\mathrm{N}), 153.3(1 \mathrm{C}), 146.1(1 \mathrm{C}), 136.7$

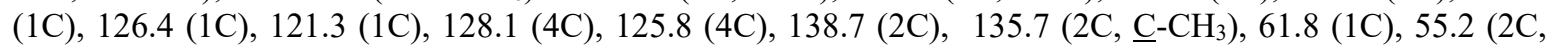
$\left.\mathrm{CH}_{3}\right), 54.5$ (1C), 46.1(1C), $36.8(1 \mathrm{C})$ ); EIMS $m / z$ (rel.int): $383[\mathrm{M}]^{+}(31), 305(27), 278(19), 265(09), 237$ (100), 161 (08), 85 (16), 73 (16), 44 (5); Anal C 78.46\%, H 6.07\%, N 14.95\%, Calcd for $\mathrm{C}_{25} \mathrm{H}_{26} \mathrm{~N}_{4}, \mathrm{C} 78.50 \%$, H $6.85 \%$, N $14.65 \%$.

(E)-4,4'-(4-((Pyridin-2-ylmethylene)hydrazono)piperidine-2,6-diyl)bis( $\mathbf{N}, \mathbf{N}$-dimethylaniline) (2g). Yellow solid: yield 86\%; mp 173-175 ${ }^{\circ} \mathrm{C}$; IR $\left(\mathrm{KBr}, \mathrm{cm}^{-1}\right)$ : 3046, 3010, 1625, 811, 726; ${ }^{1} \mathrm{H}$ NMR (DMSO-d 6$): \delta 11.28(1 \mathrm{H}, \mathrm{s}, \mathrm{NH}), 8.61(1 \mathrm{H}, \mathrm{d}, J=7.4 \mathrm{~Hz}$, pyridine $), 7.96(1 \mathrm{H}, \mathrm{s},-\mathrm{HC}=\mathrm{N}-), 7.82(1 \mathrm{H}, \mathrm{d}, J=$ $7.2 \mathrm{~Hz}$, pyridine), $7.71(1 \mathrm{H}, \mathrm{dd}, J=7.1 \mathrm{~Hz}, J=7.0 \mathrm{~Hz}$, pyridine), $7.61(1 \mathrm{H}, \mathrm{dd}, J=7.1 \mathrm{~Hz}, J=7.4 \mathrm{~Hz}$, pyridine), 7.19-7.17 (4H, d, $J=7.3 \mathrm{~Hz}$, aryl), 6.40-6.37 (4H, d, $J=7.4 \mathrm{~Hz}$, aryl), $3.69(2 \mathrm{H}, \mathrm{dd}, J=13.2 \mathrm{~Hz}, J$ $=11.4 \mathrm{~Hz}, 2 \mathrm{C}-\mathrm{H}, 6 \mathrm{C}-\mathrm{H}), 3.53(2 \mathrm{H}, \mathrm{dd}, J=13.1 \mathrm{~Hz}, J=11.4 \mathrm{~Hz}, 3 \mathrm{C}-\mathrm{Heq}, 5 \mathrm{C}-\mathrm{Heq}), 3.14\left(12 \mathrm{H}, \mathrm{s},-\mathrm{N}\left(\mathrm{CH}_{3}\right)_{2}\right)$, $2.31(2 \mathrm{H}, \mathrm{d}, J=11.22 \mathrm{~Hz}, 3 \mathrm{C}-\mathrm{Hax}, 5 \mathrm{C}-\mathrm{Hax}) ;{ }^{13} \mathrm{C}$ NMR (DMSO-d $): \delta 168.6(1 \mathrm{C}, \mathrm{C}=\mathrm{N}), 159.4(1 \mathrm{C}, \mathrm{C}=\mathrm{N})$, 153.2 (1C), 146.3 (1C), 136.1 (1C), 126.3 (1C), 121.2 (1C), 149.2 (2C), 112.9 (4C), 131.9 (4C), 133.1 (2C), $60.1(1 \mathrm{C}), 54.2(1 \mathrm{C}), 46.6(1 \mathrm{C}), 40.8\left(4 \mathrm{C},-\mathrm{N}\left(\mathrm{CH}_{3}\right)_{2}\right), 37.3(1 \mathrm{C})$; EIMS $\mathrm{m} / z$ (rel.int): $441[\mathrm{M}]^{+}$(32), 363 (28), 351 (20), 336 (19), 323 (7), 237 (100), 161(14); Anal C 73.46\%, H 7.07\%, N 19.95\%, Calcd for $\mathrm{C}_{27} \mathrm{H}_{32} \mathrm{~N}_{6}, \mathrm{C}$ $73.60 \%$, H $7.32 \%$, N $19.07 \%$.

\section{General procedure for synthesis of (E)-2-((2,6-Diphenyltetrahydro-4-thiopyran-4- ylidene)hydrazono)methyl)pyridine (4a-4g).}

A mixture of compound 3a $(0.1 \mathrm{~mol})$ and 2-(hydrazonomethyl)pyridine $(0.1 \mathrm{~mol})$ in ethanol was heated at refluxed for $2 \mathrm{~h}$. The product 4a, was purified by flash column chromatography on silica gel using hexane/EtOAc.

Yellow solid: yield $88 \%$; mp 211-214 ${ }^{\circ} \mathrm{C}$; IR $\left(\mathrm{KBr}, \mathrm{cm}^{-1}\right)$ : 760, 851,3037, 1625, 1752, 3018; ${ }^{1} \mathrm{H}$ NMR (DMSO-d $\left.\mathrm{d}_{6}\right): \delta 8.61(1 \mathrm{H}, \mathrm{d}, J=7.4 \mathrm{~Hz}$, pyridine), $7.82(1 \mathrm{H}, \mathrm{d}, J=7.2 \mathrm{~Hz}$, pyridine $), 7.78(1 \mathrm{H}, \mathrm{s},-$ $\mathrm{HC}=\mathrm{N}-), 7.71(1 \mathrm{H}, \mathrm{dd}, J=7.1 \mathrm{~Hz}, J=7.0 \mathrm{~Hz}$, pyridine $), 7.61(1 \mathrm{H}, \mathrm{dd}, J=7.1 \mathrm{~Hz}, J=7.4 \mathrm{~Hz}$, pyridine $)$, 7.45-7.29 (10H, m, aryl), $3.61(2 \mathrm{H}, \mathrm{dd}, J=13.73 \mathrm{~Hz}, J=11.5 \mathrm{~Hz}, 2 \mathrm{C}-\mathrm{H}, 6 \mathrm{C}-\mathrm{H}), 3.44(2 \mathrm{H}, \mathrm{dd}, J=13.1 \mathrm{~Hz}, J$ $=11.5 \mathrm{~Hz}, 3 \mathrm{C}-\mathrm{Heq}, 5 \mathrm{C}-\mathrm{Heq}), 2.16(2 \mathrm{H}, \mathrm{dd}, J=13.1 \mathrm{~Hz}, J=11.4 \mathrm{~Hz}, 3 \mathrm{C}-\mathrm{Hax}, 5 \mathrm{C}-\mathrm{Hax},) ;{ }^{13} \mathrm{C}$ NMR (DMSO$\left.\mathrm{d}_{6}\right): \delta 167.9(1 \mathrm{C}, \mathrm{C}=\mathrm{N}), 157.6(1 \mathrm{C}, \mathrm{C}=\mathrm{N}), 153.8(1 \mathrm{C}), 146.1(1 \mathrm{C}), 142.7-127.0(12 \mathrm{C}$, aryl), $136.0(1 \mathrm{C}), 126.3$ (1C), 121.2(1C), 60.7 (1C), 53.6 (1C), 45.9 (1C), 35.7 (1C)); EIMS m/z (rel.int): 371 [M] ${ }^{+}$(34), 294 (26), 282 (17), 267 (11), 254 (100), 102 (20), 90 (6), 61(10); Anal C 74.46\%, H 5.07\%, N 11.95\%. Calcd for $\mathrm{C}_{23} \mathrm{H}_{21} \mathrm{~N}_{3} \mathrm{~S}, \mathrm{C} 74.36 \%$, H 5.70\%, N $11.31 \%$.

(E)-2-(((2,6-Bis(4-chlorophenyl)tetrahydro-4H-thiopyran-4-lidene) hydrazono) methyl) pyridine (4b). Yellow solid: yield $82 \%$; $\mathrm{mp} 245-249^{\circ} \mathrm{C}$; IR $\left(\mathrm{KBr}, \mathrm{cm}^{-1}\right)$ : 3082, 3020, 1742, 1681, 862, 831, 671; ${ }^{1} \mathrm{H}$ NMR (DMSO-d 6 ): $\delta 8.61(1 \mathrm{H}, \mathrm{d}, J=7.4 \mathrm{~Hz}$, pyridine), $7.82(1 \mathrm{H}, \mathrm{d}, J=7.2 \mathrm{~Hz}$, pyridine $), 7.71(1 \mathrm{H}$, $\mathrm{dd}, J=7.1 \mathrm{~Hz}, J=7.0 \mathrm{~Hz}$, pyridine), $7.61(1 \mathrm{H}, \mathrm{dd}, J=7.1 \mathrm{~Hz}, J=7.4 \mathrm{~Hz}$, pyridine $), 7.56(1 \mathrm{H}, \mathrm{s},-\mathrm{HC}=\mathrm{N}-)$, 7.47-7.44 (4H, d, $J=7.3 \mathrm{~Hz}$, aryl), 7.38-7.34 $(4 \mathrm{H}, \mathrm{d}, J=7.3 \mathrm{~Hz}$, aryl), $3.79(2 \mathrm{H}, \mathrm{dd}, J=13.74 \mathrm{~Hz}, J=11.34$ $\mathrm{Hz}, 2 \mathrm{C}-\mathrm{H}, 6 \mathrm{C}-\mathrm{H}), 3.57(2 \mathrm{H}, \mathrm{dd}, J=13.30 \mathrm{~Hz}, J=11.34 \mathrm{~Hz}, 3 \mathrm{C}-\mathrm{Heq}, 5 \mathrm{C}-\mathrm{Heq}), 2.89$ (2H, dd, $J=13.34 \mathrm{~Hz}, J$ 
$=11.46 \mathrm{~Hz}, 3 \mathrm{C}-\mathrm{Hax}, 5 \mathrm{C}-\mathrm{Hax}) ;{ }^{13} \mathrm{C}$ NMR $\left(\mathrm{DMSO}^{-} \mathrm{d}_{6}\right): \delta 166.1(1 \mathrm{C}, \mathrm{C}=\mathrm{N}), 156.2(1 \mathrm{C}, \mathrm{C}=\mathrm{N}), 152.3(1 \mathrm{C})$, 147.1 (1C), 135.3 (1C), 127.9 (4C), 126.4 (1C), 121.2 (1C), 128.9 (4C), 141.3 (2C), 131.8 (2C, C-Cl), 61.8 (1C), 54.5 (1C), 46.1 (1C), 36.8 (1C); EIMS m/z (rel.int): $440[\mathrm{M}]^{+}$(32), 363 (15), 350 (21), 335 (10), 323 (12), 254 (100), 102 (13), 90 09), 61 (03); Anal C 62.46\%, H 4.07\%, N 9.95\%, Calcd for $\mathrm{C}_{23} \mathrm{H}_{19} \mathrm{Cl}_{2} \mathrm{~N}_{3} \mathrm{~S}, \mathrm{C}$ $62.73 \%, \mathrm{H} 4.35 \%$; N $9.54 \%$.

\section{(E)-4,4'-(4-((Pyridin-2-ylmethylene)hydrazono)tetrahydro-2H-thiopyran-2,6-diyl)diphenol}

(4c). Yellow solid: yield $76 \%$; mp 265-268 ${ }^{\circ} \mathrm{C}$; IR $\left(\mathrm{KBr}, \mathrm{cm}^{-1}\right)$ : 3046, 1772, 1640, 1451, 828, 614; ${ }^{1} \mathrm{H}$ NMR (DMSO-d $\left.{ }_{6}\right): \delta 9.56(2 \mathrm{H}, \mathrm{s}, \mathrm{OH}), 8.61(1 \mathrm{H}, \mathrm{d}, J=7.4 \mathrm{~Hz}$, pyridine $), 7.82(1 \mathrm{H}, \mathrm{d}, J=7.2 \mathrm{~Hz}$, pyridine), $7.71(1 \mathrm{H}, \mathrm{dd}, J=7.1 \mathrm{~Hz}, J=7.0 \mathrm{~Hz}$, pyridine), $7.61(1 \mathrm{H}, \mathrm{dd}, J=7.1 \mathrm{~Hz}, J=7.4 \mathrm{~Hz}$, pyridine $), 7.46(1 \mathrm{H}-$ $\mathrm{HC}=\mathrm{N}-), 7.19-7.17(4 \mathrm{H}, \mathrm{d}, J=7.3 \mathrm{~Hz}$, aryl), 6.70-6.65 $(4 \mathrm{H}, \mathrm{d}, J=7.3 \mathrm{~Hz}, \operatorname{aryl}), 3.74(2 \mathrm{H}, \mathrm{dd}, J=13.7 \mathrm{~Hz}, J=$ $11.6 \mathrm{~Hz}, 2 \mathrm{C}-\mathrm{H}, 6 \mathrm{C}-\mathrm{H}), 3.32(2 \mathrm{H}, \mathrm{dd}, J=13.8 \mathrm{~Hz}, J=11.6 \mathrm{~Hz}, 3 \mathrm{C}-\mathrm{Heq}, 5 \mathrm{C}-\mathrm{Heq}), 2.24(2 \mathrm{H}, \mathrm{dd}, J=13.8 \mathrm{~Hz}$, $J=11.3 \mathrm{~Hz}, 3 \mathrm{C}-\mathrm{Hax}, 5 \mathrm{C}-\mathrm{Hax}, 1 \mathrm{H}) ;{ }^{13} \mathrm{C}$ NMR $\left(\mathrm{DMSO}_{\mathrm{d}}\right): \delta 168.3(1 \mathrm{C}, \mathrm{C}=\mathrm{N}), 159.2(2 \mathrm{C}, \mathrm{C}-\mathrm{OH}), 156.4(1 \mathrm{C}$, $\mathrm{C}=\mathrm{N}), 153.0$ (1C), $146.2(1 \mathrm{C}), 136.7$ (1C), 136.3 (2C), 127.9 (4C), 126.4 (1C), $121.1(1 \mathrm{C}), 115.6(4 \mathrm{C}), 62.6$ (1C), 55.8 (1C), 46.1 (1C), 36.8 (1C); EI MS m/z (rel.int): 403 [M] $]^{+}$(47), 326 (16), 314 (23), 299 (11), 286 (23), 254 (100), 102 (16), 90 (7), 61 (2); Anal C 68.46\%, H 5.07\%, N 10.95\%, Calcd for $\mathrm{C}_{23} \mathrm{H}_{21} \mathrm{~N}_{3} \mathrm{O}_{2} \mathrm{~S}, \mathrm{C}$ $68.46 \%$, H $5.25 \%$, N $10.41 \%$.

(E)-2-((2,6-Bis(4-nitrophenyl)tetrahydro-4H-thiopyran-4-ylidene)hydrazono)methyl)pyridine (4d). Yellow solid: yield $86 \%$; mp 255-258 ${ }^{\circ} \mathrm{C}$; IR $\left(\mathrm{KBr}, \mathrm{cm}^{-1}\right): 3079,3021,1711,1684,1531,808,651 ;{ }^{1} \mathrm{H}$ NMR (DMSO-d 6 ): $\delta 8.61(1 \mathrm{H}, \mathrm{d}, J=7.4 \mathrm{~Hz}$, pyridine), 8.45-8.40 (4H, d, $J=7.8 \mathrm{~Hz}$, aryl), $7.82(1 \mathrm{H}, \mathrm{d}, J=7.2$ $\mathrm{Hz}$, pyridine), $7.71(1 \mathrm{H}, \mathrm{dd}, J=7.1 \mathrm{~Hz}, J=7.0 \mathrm{~Hz}$, pyridine), $7.61(1 \mathrm{H}, \mathrm{dd}, J=7.1 \mathrm{~Hz}, J=7.4 \mathrm{~Hz}$, pyridine), $7.40(1 \mathrm{H}, \mathrm{s},-\mathrm{HC}=\mathrm{N}-), 7.29-7.27(4 \mathrm{H}, \mathrm{d}, J=7.8 \mathrm{~Hz}, \operatorname{aryl}), 3.79(2 \mathrm{H}, \mathrm{dd}, J=13.70 \mathrm{~Hz}, J=11.67 \mathrm{~Hz}, 2 \mathrm{C}-\mathrm{H}$, $6 \mathrm{C}-\mathrm{H}), 3.35(2 \mathrm{H}, \mathrm{dd}, J=13.68 \mathrm{~Hz}, J=11.67 \mathrm{~Hz}, 3 \mathrm{C}-\mathrm{Heq}, 5 \mathrm{C}-\mathrm{Heq}), 2.11(2 \mathrm{H}, \mathrm{dd}, J=13.67 \mathrm{~Hz}, J=11.39$ $\mathrm{Hz}, 3 \mathrm{C}-\mathrm{Hax}, 5 \mathrm{C}-\mathrm{Hax}) ;{ }^{13} \mathrm{C}$ NMR (DMSO-d $): \delta 167.1(1 \mathrm{C}, \mathrm{C}=\mathrm{N}), 157.7(1 \mathrm{C}, \mathrm{C}=\mathrm{N}), 153.2(1 \mathrm{C}), 148.3(2 \mathrm{C})$, 147.2 (1C), $145.2\left(2 \mathrm{C}-\mathrm{NO}_{2}\right), 136.1$ (1C), 127.0 (1C), 124.9 (4C), 124.6 (4C), $121.3(1 \mathrm{C}), 61.8(1 \mathrm{C}), 54.5$ (1C), 46.1 (1C), 36.8 (1C); EI MS m/z (rel.int): $461[\mathrm{M}]^{+}$(51), 382 (35), 357 (18), 344 (28), 254 (100), 102 (28), 90 (14), 61 (7); Anal C 59.46\%, H 5.07\%, N 15.95\%, S 6.90, Calcd for $\mathrm{C}_{23} \mathrm{H}_{19} \mathrm{~N}_{5} \mathrm{O}_{4} \mathrm{~S}, \mathrm{C} 59.86 \%, \mathrm{H}$ $4.15 \%, \mathrm{~N} 15.18 \%$, S $6.98 \%$.

\section{(E)-2-((2,6-Bis(4-methoxyphenyl)tetrahydro-4H-thiopyran-4-ylidene)hydrazono)methyl)}

pyridine (4e). Yellow solid: yield $89 \%$; $\mathrm{mp} 272-277^{\circ} \mathrm{C}$; $\mathrm{IR}\left(\mathrm{KBr}, \mathrm{cm}^{-1}\right): 3085,1732,1671,806,661 ;{ }^{1} \mathrm{H}$ NMR (DMSO-d 6 ): $\delta 8.61(1 \mathrm{H}, \mathrm{d}, J=7.4 \mathrm{~Hz}$, pyridine), $7.82(1 \mathrm{H}, \mathrm{d}, J=7.2 \mathrm{~Hz}$, pyridine $), 7.71(1 \mathrm{H}, \mathrm{dd}, J$ $=7.1 \mathrm{~Hz}, J=7.0 \mathrm{~Hz}$, pyridine), $7.61(1 \mathrm{H}, \mathrm{dd}, J=7.1 \mathrm{~Hz}, J=7.4 \mathrm{~Hz}$, pyridine), 7.22-7.20 (4H, d, $J=7.1 \mathrm{~Hz}$, aryl), 6.35-6.32 $(4 \mathrm{H}, \mathrm{d}, J=7.1 \mathrm{~Hz}$, aryl $), 7.43(1 \mathrm{H}, \mathrm{s},-\mathrm{HC}=\mathrm{N}-), 3.85\left(6 \mathrm{H}, \mathrm{s},-\mathrm{OCH}_{3}\right), 3.72(2 \mathrm{H}, \mathrm{dd}, J=13.9$ $\mathrm{Hz}, J=11.6 \mathrm{~Hz}, 2 \mathrm{C}-\mathrm{H}, 6 \mathrm{C}-\mathrm{H}), 3.49(2 \mathrm{H}, \mathrm{d}, J=11.6 \mathrm{~Hz}, 3 \mathrm{C}-\mathrm{Heq}, 5 \mathrm{C}-\mathrm{Heq}), 2.17(2 \mathrm{H}, \mathrm{dd}, J=13.8 \mathrm{~Hz}, J=$ $11.4 \mathrm{~Hz}, 3 \mathrm{C}-\mathrm{Hax}, 5 \mathrm{C}-\mathrm{Hax}) ;{ }^{13} \mathrm{C}$ NMR(DMSO-d 6$): \delta 168.2(1 \mathrm{C}, \mathrm{C}=\mathrm{N}), 158.3(1 \mathrm{C}, \mathrm{C}=\mathrm{N}), 157.9(2 \mathrm{C}, \underline{\mathrm{C}}-$ $\left.\mathrm{OCH}_{3}\right), 154.2(1 \mathrm{C}), 147.0(1 \mathrm{C}), 135.8(2 \mathrm{C}), 135.2(1 \mathrm{C}), 127.1(1 \mathrm{C}), 121.4(1 \mathrm{C}), 114.3(4 \mathrm{C}), 126.4(4 \mathrm{C}), 61.8$ (1C), 55.8 (2C, $\left.-\mathrm{OCH}_{3}\right), 54.5$ (1C), 46.1 (1C), 36.8 (1C); EIMS m/z (rel.int): 432 [M] ${ }^{+}$(49), 354 (26), 327 (54), 314 (24), 254 (100), 102 (28), 90 (17), 61 (8); Anal C 69.56\%, H 5.87\%, N 9.75\%, S 7.48\%, Calcd for $\mathrm{C}_{25} \mathrm{H}_{25} \mathrm{~N}_{3} \mathrm{O}_{2} \mathrm{~S}$, C 69.58\%; H, 5.84\%; N, 9.74\%; S, 7.43\%.

(E)-2-((2,6-Di-p-tolyltetrahydro-4H-thiopyran-4-ylidene)hydrazono)methyl)pyridine

(4f). Yellow solid: yield $84 \%$; mp 258-261 ${ }^{\circ} \mathrm{C}$; IR $\left(\mathrm{KBr}, \mathrm{cm}^{-1}\right): 3094,1762,1651,825,671 ;{ }^{1} \mathrm{H}$ NMR (DMSO-d 6 ): $\delta 8.61(1 \mathrm{H}, \mathrm{d}, J=7.4 \mathrm{~Hz}$, pyridine), $7.82(1 \mathrm{H}, \mathrm{d}, J=7.2 \mathrm{~Hz}$, pyridine), $7.71(1 \mathrm{H}, \mathrm{dd}, J=7.1 \mathrm{~Hz}, J=7.0 \mathrm{~Hz}$, pyridine), $7.61(1 \mathrm{H}, \mathrm{dd}, J=7.1 \mathrm{~Hz}, J=7.4 \mathrm{~Hz}$, pyridine), $7.48(1 \mathrm{H}, \mathrm{s},-\mathrm{HC}=\mathrm{N}-), 7.45-7.41(4 \mathrm{H}, \mathrm{d}, J=7.4 \mathrm{~Hz}$, aryl), 7.29-7.25 (4H, d, $J=7.4 \mathrm{~Hz}$, aryl), $3.72(2 \mathrm{H}, \mathrm{dd}, J=13.7 \mathrm{~Hz}, J=11.3 \mathrm{~Hz}, 2 \mathrm{C}-\mathrm{H}, 6 \mathrm{C}-\mathrm{H}), 3.36(2 \mathrm{H}$, dd, $J=13.5 \mathrm{~Hz}, J=11.3 \mathrm{~Hz}, 3 \mathrm{C}-\mathrm{Heq}, 5 \mathrm{C}-\mathrm{Heq}), 2.28\left(6 \mathrm{H}, \mathrm{s}, \mathrm{CH}_{3}\right), 2.08(2 \mathrm{H}, \mathrm{dd}, J=13.6 \mathrm{~Hz}, J=11.3 \mathrm{~Hz}, 3 \mathrm{C}-$ Hax, 5C-Hax); ${ }^{13} \mathrm{C}$ NMR(DMSO-d $): \delta 167.1(1 \mathrm{C}, \mathrm{C}=\mathrm{N}), 157.6(1 \mathrm{C}, \mathrm{C}=\mathrm{N}), 153.1(1 \mathrm{C}), 146.0(1 \mathrm{C}), 138.7$ (2C), 136.1 (1C), 135.7 (2C, Ph-CH $), 128.1$ (4C), 126.4 (1C), 125.8 (4C), 121.2 (1C), 61.8 (1C), 54.5 (1C), 46.1 (1C), 36.8 (1C), 21.7 (2C-CH $)$; EI MS m/z (rel.int): $\left.400[\mathrm{M}]^{+} 32\right), 322$ (37), 295 (21), 282 (19), 254 (100), 102 (36), 90 (18), 61 (11); Anal C 75.18\%, H 6.37\%, N 10.55\%, S 8.10\%, Calcd for $\mathrm{C}_{25} \mathrm{H}_{25} \mathrm{~N}_{3} \mathrm{~S}, \mathrm{C}$ $75.15 \%$; H $6.31 \%$, N $10.52 \%$ S $8.03 \%$. 
(E)-4,4'-(4-((Pyridin-2-ylmethylene)hydrazono)tetrahydro-2H-thiopyran-2,6-diyl)bis $(\mathrm{N}, \mathrm{N}$ dimethylaniline) (4g). Yellow solid: yield $88 \%$; mp 268-270 ${ }^{\circ} \mathrm{C}$; $\mathrm{IR}\left(\mathrm{KBr}, \mathrm{cm}^{-1}\right): 3046,1782,1625,811$, 664; ${ }^{1} \mathrm{H}$ NMR (DMSO-d 6 ): $\delta 8.61(1 \mathrm{H}, \mathrm{d}, J=7.4 \mathrm{~Hz}$, pyridine), $7.82(1 \mathrm{H}, \mathrm{d}, J=7.2 \mathrm{~Hz}$, pyridine), $7.71(1 \mathrm{H}$, dd, $J=7.1 \mathrm{~Hz}, J=7.0 \mathrm{~Hz}$, pyridine), $7.61(1 \mathrm{H}, \mathrm{dd}, J=7.1 \mathrm{~Hz}, J=7.4 \mathrm{~Hz}$, pyridine), $7.46(1 \mathrm{H}, \mathrm{s},-\mathrm{HC}=\mathrm{N}-)$, 7.23-7.19 $(4 \mathrm{H}, \mathrm{d}, J=7.1 \mathrm{~Hz}$, aryl ), 6.48-6.42 $(4 \mathrm{H}, \mathrm{d}, J=7.2 \mathrm{~Hz}$, aryl ), $3.69(2 \mathrm{H}, \mathrm{dd}, J=13.2 \mathrm{~Hz}, J=11.4$ $\mathrm{Hz}, 2 \mathrm{C}-\mathrm{H}, 6 \mathrm{C}-\mathrm{H}), 3.53(2 \mathrm{H}, \mathrm{dd}, J=13.1 \mathrm{~Hz}, J=11.4 \mathrm{~Hz}, 3 \mathrm{C}-\mathrm{Heq}, 5 \mathrm{C}-\mathrm{Heq}), 3.14\left(12 \mathrm{H}, \mathrm{s},-\mathrm{N}\left(\mathrm{CH}_{3}\right)_{2}\right), 2.31$ $(2 \mathrm{H}, \mathrm{d}, J=11.4 \mathrm{~Hz}, 3 \mathrm{C}-\mathrm{Hax}, 5 \mathrm{C}-\mathrm{Hax}) ;{ }^{13} \mathrm{C}$ NMR $\left(\right.$ DMSO-d $\left._{6}\right): \delta 168.6(1 \mathrm{C}, \mathrm{C}=\mathrm{N}), 159.4(1 \mathrm{C}, \mathrm{C}=\mathrm{N}), 153.8$ (1C), 148.2 (2C, $\left.\underline{\mathrm{C}}-\mathrm{N}\left(\mathrm{CH}_{3}\right)_{2}\right), 146.1$ (1C), 136.3 (1C), 133.1 (2C), 131.9 (4C), 126.6 (1C), 121.2 (1C), 112.9 (4C), $60.1(1 \mathrm{C}), 54.2(1 \mathrm{C}), 46.6(1 \mathrm{C}), 40.8$ (4C, Ph-N $\left.\left(\mathrm{CH}_{3}\right)_{2}\right), 37.3$ (1C); EIMS $\mathrm{m} / z$ (rel.int): $458[\mathrm{M}]^{+}(16)$, 381 (43), 354 (24), 341 (17), 254 (100), 102 (21), 90 (10), 61 (3); Anal C 70.46\%, H 6.07\%, N 15.95\%, Calcd for $\mathrm{C}_{27} \mathrm{H}_{31} \mathrm{~N}_{5} \mathrm{~S}, \mathrm{C} 70.86 \%$, H $6.83 \%$, N $15.30 \%$.

\section{Larvicidal activity}

Larvicidal screening was performed following the methodology described in our previous study [22]. Synthesized compounds were tested against the urban mosquito larvae, Culex quinquefasciatus. Eggs of $C$. quinquefasciatus were obtained from the city drainage system. Eggs were placed in clean water and kept at room temperature for hatching. Larval development was monitored for 7 days. Second stage larvae were collected using a pasture pipette, placed in cotton to remove excess water, and transferred to test vials. Larval mortality was observed using increasing concentrations of synthesized compounds $(10,20,30$, and 40 $\mu \mathrm{g} / \mathrm{mL}$ ). The susceptibility or resistance of the mosquito larvae to the selected concentration of the synthesized compounds was determined with a standard protocol (WHO, 1981).

\section{Nematicidal activity}

Nematicidal activity was evaluated using juvenile nematodes of Meloidogyne javanica [22]. The assay system was prepared with $2 \mathrm{~mL}$ Milli-Q ${ }^{\circledR}$ water, containing different concentrations of compound (10, 20,30 , and $40 \mu \mathrm{g} / \mathrm{mL}$ ) in glass tubes. Treated and control nematodes were held under the same conditions used for colony maintenance. Ten nematode juveniles were transferred into each tube. Positive (levamisole) and negative ( $2 \%$ DMSO) control tubes were included. Mortality was observed under a zoom stereomicroscope after $24 \mathrm{~h}$ of exposure. The mortality percentage was converted into probit scale to determine the $\mathrm{LD}_{50}$ values.

\section{In vitro antimicrobial screening}

Antimicrobial activity: The compounds 2a-g, 3a-g, and 4a-g were inspected for their in vitro antibacterial activity against a battery of human type culture pathogens such as Staphylococcus aureus (ATCC-25923), Klebsiella pneumoniae, Escherichia coli (ATCC-25922), and Pseudomonas aeruginosa (ATCC-27853) by disc diffusion method [23] using Mueller-Hinton broth (Hi-media). The same compounds were evaluated for in vitro antifungal activity against a panel of human fungal pathogens such as Aspergillus niger, Candida albicans, Aspergillus fumigatus, Cryptococcus neoformans and Microsporum audouinii using an broth dilution method [24] in Sabouraud's dextrose broth (Hi-Media).

In order to determine the minimum inhibitory concentration (MIC), tube dilution method was used with respective compounds aliquoted in phosphate-buffered saline $(\mathrm{pH} 7.2)$ as test solution. Briefly, the dosing range of compounds were calculated by the factor 2 (anti $\log 0.3$ ) in order to obtain a dose range between 0.5 to $128 \mu \mathrm{g}$ per $\mathrm{mL}$ in Mueller Hinton broth. Afterwards, respective tubes were inoculated with $100 \mu \mathrm{L}$ of fresh culture of appropriate bacterial and fungal pathogens $\left(10^{4}\right.$ to $\left.10^{5} \mathrm{CFU} / \mathrm{mL}\right)$ and incubated at $37 \pm 2{ }^{\circ} \mathrm{C}$ for $24-$ $72 \mathrm{~h}$, respectively. MICs were recorded as the lowest concentrations inhibiting visible growth of the microorganisms as compared to the negative controls.

\section{Statistical analysis}

All the experiments were repeated three times to validate the findings statistically [25]. All the data are presented as mean \pm standard deviation (S.D.). Mean values were compared among treatments and the control using one way analysis of variance (ANOVA) using SPSS at $P<0.05$ levels. 


\section{Results and discussion}

Chemistry. Compounds 1a-g were synthesized according to the method described previously [26]. Compounds 3a-g were prepared by the method reported elsewhere [27-28]. Compounds 2a-g and 4a-g were synthesized by condensation method (Scheme 1). The physicochemical data of compounds 2a-g and 4a-g are shown in experimental section. The formation of all the compounds was confirmed by recording the IR, ${ }^{1} \mathrm{H}$ NMR, ${ }^{13} \mathrm{C}$ NMR spectra, and elemental analyses. The IR spectra of compounds 2a-g showed absorption bands at $3045-3094$, and $1625-1684 \mathrm{~cm}^{-1}$ corresponding to the $\mathrm{NH}$ and $\mathrm{C}=\mathrm{N}$ groups, respectively. The ${ }^{1} \mathrm{H}$ NMR spectra of compounds 2a-g showed a sharp singlet at $\delta 11.15-11.71$ for NH proton and a singlet at $\delta$ 7.48-7.96 for $\mathrm{HC}=\mathrm{N}-$ proton. The ${ }^{13} \mathrm{C}$ NMR spectra of compounds $\mathbf{2 a - g}$ showed characteristic peaks at $\delta$ 156.2-164.2 and $\delta 166.1-168.7 \mathrm{ppm}$ corresponding to $\mathrm{C}=\mathrm{N}$ and $-\mathrm{HC}=\mathrm{N}-$ carbons, respectively. The IR spectra of compounds 4a-g showed absorption bands at 1711-1782, 1625-1684, and 614-760 $\mathrm{cm}^{-1}$ corresponding to $\mathrm{C}=\mathrm{N}, \mathrm{HC}=\mathrm{N}$, and $\mathrm{C}-\mathrm{S}-\mathrm{C}$ groups, respectively. The ${ }^{1} \mathrm{H}$ NMR spectra of compounds $\mathbf{4 a - g}$ showed signals at $\delta 7.40-7.78$, which confirmed the presence of $\mathrm{HC}=\mathrm{N}$ - proton. The ${ }^{13} \mathrm{C}$ NMR spectra of compounds 4a-g showed characteristic peaks at $\delta 166.1-168.6$ and $\delta 156.2-159.4$ ppm corresponding to $\mathrm{C}=\mathrm{N}$ and $-\mathrm{HC}=\mathrm{N}$ - carbons, respectively. In addition, mass spectra showed that the molecular ion signals matched the expected molecular weights of all the synthesized compounds.

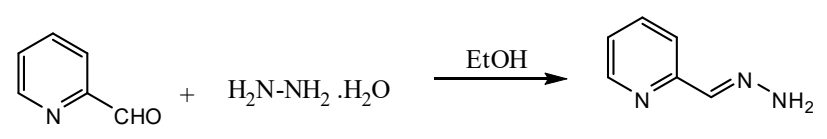

Synthesis of 2-(hydrazonomethyl)pyridine (HMP)
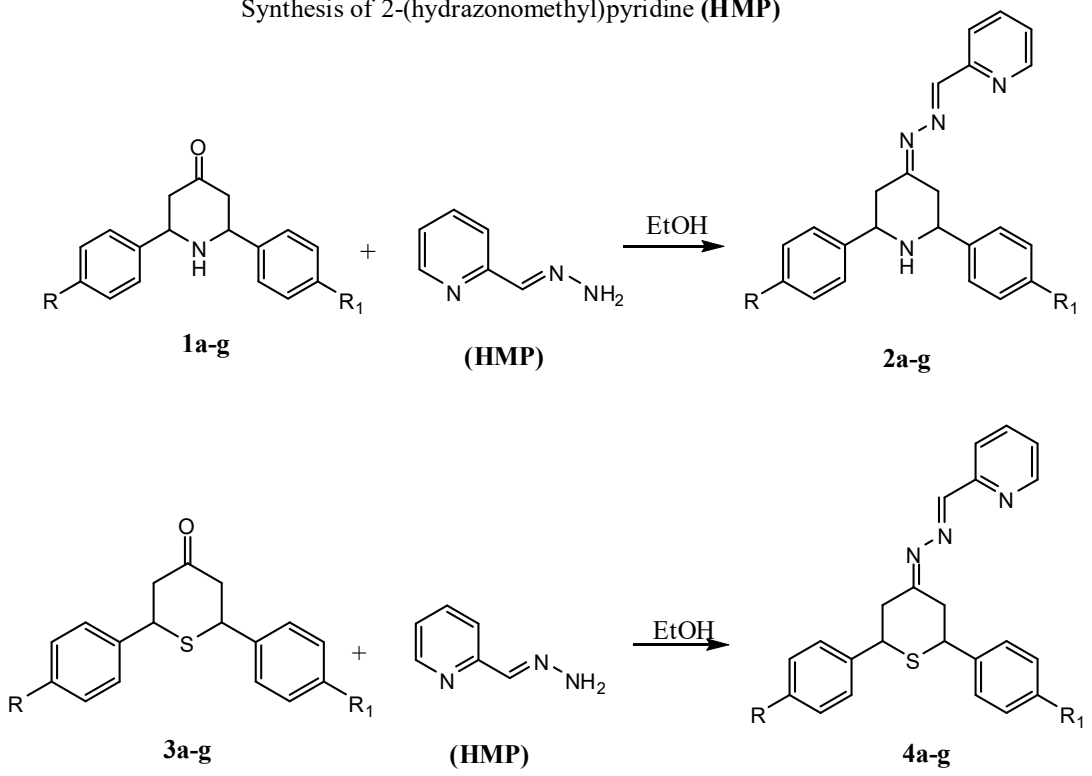

\begin{tabular}{|l|}
\hline 1a, 2a, 3a, 4a: R, $\mathrm{R}_{1}=-\mathrm{H}$ \\
\hline 1b, 2b, 3b,4b: R, $\mathrm{R}_{1}=-\mathrm{Cl}$ \\
\hline 1c, 2c, 3c,4c: R, $\mathrm{R}_{1}=-\mathrm{HO}$ \\
\hline 1d, 2d, 3d, 4d: R, $\mathrm{R}_{1}=-\mathrm{NO}_{2}$ \\
\hline 1e, 2e, 3e, 4e: $\mathrm{R}, \mathrm{R}_{1}=-\mathrm{OCH}_{3}$ \\
\hline 1f, 2f, 3f, 4f: $\mathrm{R}, \mathrm{R}_{1}=-\mathrm{CH}_{3}$ \\
\hline 1g, 2g, 3g, 4g: R, $\mathrm{R}_{1}=-\mathrm{N}\left(\mathrm{CH}_{3}\right)_{2}$ \\
\hline
\end{tabular}

Scheme 1. Synthesis of 2-thio-imidazolidin-4-one derivatives 2a-g and 4a-g 
Larvicidal activity. Compounds $\mathbf{2 a - g}$ and $\mathbf{4 a - g}$ were screened for larvicidal activity. Compounds $\mathbf{2 a - g}$ exhibited lower rank of larvicidal activity than compounds 4a-g. Compound 4e showed higher rank of larvicidal activity than other compounds and produced $100 \%$ mortality $(20 \mu \mathrm{g} / \mathrm{mL})$, with corresponding LD50 value of $0.8 \mu \mathrm{g} / \mathrm{mL}$. Compounds $\mathbf{4 c}, \mathbf{4 d}, \mathbf{4 f}$, and $\mathbf{4 g}$ showed moderate activity with concomitant $\mathrm{LD}_{50}$ values of 5.7, 1.2, 8.6, and $6.0 \mu \mathrm{g} / \mathrm{mL}$, respectively. The values are summarized in Table 1 .

Table 1. Larvicidal profile of compounds (2a-g and 4a-g) on second instar larvae of Culex sp.

\begin{tabular}{|c|c|c|c|c|c|}
\hline \multirow[t]{3}{*}{ Comp.No } & \multicolumn{4}{|c|}{ Mortality (\%)Room temp } & \multirow{3}{*}{$\begin{array}{c}\text { LD }_{50} \\
(\mu \mathrm{g} / \mathrm{mL})\end{array}$} \\
\hline & \multicolumn{4}{|c|}{ Concentration $(\mu \mathrm{g} / \mathrm{mL})^{\mathrm{a}}$} & \\
\hline & 10 & 20 & 30 & 40 & \\
\hline $2 a$ & $30.4 \pm 1.2$ & $64.5 \pm 1.5$ & $72.3 \pm 0.4$ & $100 \pm 0.0$ & 17.4 \\
\hline $2 \mathbf{b}$ & $50.3 \pm 1.7$ & $66.3 \pm 1.4$ & $82.5 \pm 1.0$ & $100 \pm 0.0$ & 10.2 \\
\hline $2 c$ & $39.2 \pm 1.3$ & $63.9 \pm 1.0$ & $78.0 \pm 0.7$ & $100 \pm 0.0$ & 16.8 \\
\hline 2d & $40.9 \pm 1.8$ & $61.3 \pm 1.2$ & $84.7 \pm 1.0$ & $100 \pm 0.0$ & 15.7 \\
\hline $2 e$ & $32.2 \pm 1.3$ & $42.5 \pm 1.1$ & $69.4 \pm 1.2$ & $85.6 \pm 2.1$ & 24.3 \\
\hline $2 f$ & $41.1 \pm 0.7$ & $51.4 \pm 1.0$ & $60.5 \pm 1.0$ & $82.4 \pm 1.1$ & 19.5 \\
\hline $2 g$ & $32.0 \pm 0.6$ & $47.4 \pm 1.6$ & $81.6 \pm 0.5$ & $100 \pm 0.0$ & 21.3 \\
\hline $4 \mathbf{a}$ & $31.5 \pm 0.8$ & $62.0 \pm 0.5$ & $100 \pm 0.0$ & - & 16.6 \\
\hline $4 b$ & $20.4 \pm 0.3$ & $40.4 \pm 1.0$ & $56.8 \pm 0.0$ & $75.7 \pm 0.3$ & 26.5 \\
\hline $4 \mathrm{c}$ & $62.4 \pm 1.3$ & $81.5 \pm 1.4$ & $100 \pm 0.0$ & - & 5.7 \\
\hline 4d & $80.3 \pm 1.4$ & $100 \pm 0.0$ & - & - & 1.2 \\
\hline $4 \mathrm{e}$ & $88.1 \pm 1.9$ & $100 \pm 0.0$ & - & - & 0.8 \\
\hline $4 f$ & $57.4 \pm 0.5$ & $84.7 \pm 1.0$ & $100 \pm 0.0$ & - & 8.6 \\
\hline $4 g$ & $61.3 \pm 1.1$ & $78.0 \pm 1.1$ & $100 \pm 0.0$ & - & 6.0 \\
\hline Positive control & $43.1 \pm 0.3$ & $56.7 \pm 0.1$ & $61.8 \pm 1.1$ & $100 \pm 0.0$ & 15.2 \\
\hline Negative control & $0.0 \pm 0.0$ & $0.0 \pm 0.0$ & $0.0 \pm 0.0$ & $0.0 \pm 0.0$ & \\
\hline
\end{tabular}

${ }^{a}$ Values are the means of three replicates $\pm \mathrm{SD}$.

Positive control: $N$-tert-butyl- $N, N^{\prime}$ - dibenzoylhydrazine;

Negative control: Dimethyl sulfoxide (DMSO)

Nematicidal activity. Compounds 2a-g and 4a-g were inspected for nematicidal activity, and compounds 2a-g exhibited lower range of nematicidal activity than compounds 4a-g. The screening was carried out at $37^{\circ} \mathrm{C}$ and the toxicity of the compounds were measured. Compound $4 \mathrm{e}$ showed higher degree of nematicidal activity than other compounds and produced $100 \%$ mortality at $20 \mu \mathrm{g} / \mathrm{mL}$, with corresponding $\mathrm{LD}_{50}$ value of $3.2 \mu \mathrm{g} / \mathrm{mL}$. Compounds $\mathbf{4 c}, \mathbf{4 d}, \mathbf{4 f}$, and $\mathbf{4 g}$ showed moderate range of activities with $\mathrm{LD}_{50}$ values of 7.8, 5.5, 4.1 , and $4.7 \mu \mathrm{g} / \mathrm{mL}$, respectively. The values are summarized in Table 2 . 
Table 2. Nematicidal activity of synthesized compounds (2a-g and 4a-g)

\begin{tabular}{|c|c|c|c|c|c|}
\hline \multirow{3}{*}{ Comp.No. } & \multicolumn{4}{|c|}{ Mortality (\%)Room temp } & \multirow{3}{*}{$\begin{array}{c}\mathbf{L D}_{50} \\
(\mu \mathrm{g} / \mathrm{mL})\end{array}$} \\
\hline & \multicolumn{4}{|c|}{ Concentration $(\mu \mathrm{g} / \mathrm{mL})^{\mathrm{a}}$} & \\
\hline & 10 & 20 & 30 & 40 & \\
\hline $2 \mathbf{a}$ & $48.3 \pm 3.5$ & $62.0 \pm 2.9$ & $74.4 \pm 2.4$ & $88.0 \pm 0.6$ & 11.8 \\
\hline $2 b$ & $40.2 \pm 1.6$ & $57.6 \pm 2.0$ & $72.2 \pm 1.7$ & $85.0 \pm 0.0$ & 15.5 \\
\hline $2 c$ & $36.1 \pm 2.0$ & $59.5 \pm 3.1$ & $64.7 \pm 2.0$ & $100 \pm 0.0$ & 17.6 \\
\hline $2 d$ & $48.8 \pm 4.4$ & $63.0 \pm 3.0$ & $81.0 \pm 3.6$ & $100 \pm 0.0$ & 11.2 \\
\hline $2 e$ & $41.0 \pm 3.1$ & $59.2 \pm 1.6$ & $80 \pm 1.0$ & $100 \pm 0.0$ & 17.7 \\
\hline $2 f$ & $48.6 \pm 2.0$ & $60.2 \pm 3.1$ & $100 \pm 0.0$ & - & 12.5 \\
\hline $2 g$ & $44.5 \pm 4.2$ & $69.1 \pm 1.9$ & $81.9 \pm 2.9$ & $100 \pm 0.0$ & 20.0 \\
\hline $4 \mathbf{a}$ & $54.1 \pm 2.1$ & $66.7 \pm 2.1$ & $81.3 \pm 1.5$ & $100 \pm 0.0$ & 8.4 \\
\hline $4 \mathbf{b}$ & $51.0 \pm 1.9$ & $72.6 \pm 2.5$ & $84.5 \pm 1.0$ & $100 \pm 0.0$ & 9.5 \\
\hline $4 c$ & $56.7 \pm 0.3$ & $62.0 \pm 1.2$ & $100 \pm 0.0$ & - & 7.8 \\
\hline 4d & $61.3 \pm 2.5$ & $87.6 \pm 1.0$ & $100 \pm 0.0$ & - & 5.5 \\
\hline $4 e$ & $87.8 \pm 2.5$ & $100 \pm 0.0$ & - & - & 3.2 \\
\hline $4 f$ & $72.4 \pm 2.5$ & $83.8 \pm 2.5$ & $100 \pm 0.0$ & - & 4.1 \\
\hline $4 \mathrm{~g}$ & $69.2 \pm 2.5$ & $86.5 \pm 2.5$ & $100 \pm 0.0$ & - & 4.7 \\
\hline Positive control & $40.9 \pm 1.1$ & $57.8 \pm 1.2$ & $80.2 \pm 1.1$ & $100 \pm 0.0$ & 14.2 \\
\hline Negative control & $0.0 \pm 0.0$ & $0.0 \pm 0.0$ & $0.0 \pm 0.0$ & $0.0 \pm 0.0$ & \\
\hline
\end{tabular}

${ }^{\text {a }}$ Values are the means of three replicates \pm SD

Positive control: (-)-Pinidinol;

Negative control: Dimethyl sulfoxide (DMSO)

Antibacterial activity. The synthesized compounds 2a-g, and 4a-g were evaluated for antibacterial activity against various human bacterial pathogens. Compound 2d (MIC: $4 \mu \mathrm{g} / \mathrm{mL}$ ) showed high antibacterial activity against $E$. coli. Compound 2e (MIC: $4 \mu \mathrm{g} / \mathrm{mL}$ ) showed higher antibacterial activity against $K$. pneumoniae than ciprofloxacin (MIC: $16 \mu \mathrm{g} / \mathrm{mL}$ ). The MIC values are summarized in Table 3 .

Table 3. Antibacterial screening: Minimum inhibitory concentration (MIC) in $\mu \mathrm{g} / \mathrm{mL}$

\begin{tabular}{|c|c|c|c|c|}
\hline \multirow[t]{2}{*}{ Compounds } & \multirow{2}{*}{$\begin{array}{c}\text { Gram-positive } \\
\text { S. aureus }\end{array}$} & \multicolumn{3}{|c|}{ Gram-negative } \\
\hline & & E. coli & P. aeruginosa & K. pneumoniae \\
\hline $2 a$ & 32 & $>100$ & $>100$ & 64 \\
\hline $2 b$ & 32 & 32 & 32 & 32 \\
\hline $2 c$ & 32 & 32 & 32 & 32 \\
\hline 2d & 4 & 4 & 8 & 64 \\
\hline $2 \mathrm{e}$ & 8 & 64 & $>100$ & 4 \\
\hline $2 f$ & 32 & 32 & 32 & 32 \\
\hline $2 \mathrm{~g}$ & 4 & 16 & 16 & 32 \\
\hline $4 a$ & 64 & $>100$ & $>100$ & $>100$ \\
\hline $4 b$ & 64 & $>100$ & $>100$ & $>100$ \\
\hline $4 c$ & 64 & $>100$ & $>100$ & $>100$ \\
\hline 4d & 64 & $>100$ & $>100$ & 64 \\
\hline $4 \mathrm{e}$ & 64 & 64 & 64 & 64 \\
\hline 4f & 64 & 64 & 64 & 64 \\
\hline $4 \mathrm{~g}$ & 64 & 64 & 64 & 64 \\
\hline Ciprofloxacin & 4 & 8 & 8 & 16 \\
\hline
\end{tabular}


Antifungal activity. Compounds $\mathbf{2 a - g}$ and $\mathbf{4 a - g}$ were screened for antifungal activity against various fungal pathogens. Compound 4b (MIC: $0.25 \mu \mathrm{g} / \mathrm{mL}$ ) exhibited high antifungal activity against $C$. albicans whereas the compound $4 \mathrm{e}$ (MIC: $0.5 \mu \mathrm{g} / \mathrm{mL}$ ) showed slight equipotent activity against $C$. albicans. Compound $4 \mathrm{f}$ (MIC: $2 \mu \mathrm{g} / \mathrm{mL}$ ) showed higher activity against $M$. audouinii than clotrimazole (MIC: $4 \mu \mathrm{g} / \mathrm{mL}$ ). The antifungal activity values are appended in Table 4.

Table 4. Antifungal screening: Minimum inhibitory concentration (MIC) in $\mu \mathrm{g} / \mathrm{mL}$

\begin{tabular}{|c|c|c|c|c|}
\hline Compounds & A. niger & C. albicans & M. audouinii & C. neoformans \\
\hline $\mathbf{2 a}$ & $>100$ & $>100$ & $>100$ & $>100$ \\
\hline $\mathbf{2 b}$ & 32 & 4 & 32 & $>100$ \\
\hline $\mathbf{2 c}$ & $>100$ & $>100$ & $>100$ & $>100$ \\
\hline $\mathbf{2 d}$ & 64 & $>100$ & $>100$ & $>100$ \\
\hline $\mathbf{2 e}$ & 64 & $>100$ & $>100$ & $>100$ \\
\hline $\mathbf{2 f}$ & 64 & 64 & $>100$ & $>100$ \\
\hline $\mathbf{2 g}$ & $>100$ & 64 & $>100$ & $>100$ \\
\hline $\mathbf{4 a}$ & $>100$ & 32 & $>100$ & 32 \\
\hline $\mathbf{4 b}$ & $>100$ & 0.25 & 32 & 16 \\
\hline $\mathbf{4 c}$ & 16 & 32 & $>100$ & 16 \\
\hline $\mathbf{4 d}$ & 16 & 8 & 64 & $>100$ \\
\hline $\mathbf{4 e}$ & 16 & 0.5 & $>100$ & 64 \\
\hline $\mathbf{4 f}$ & 32 & 32 & 2 & 8 \\
\hline $\mathbf{4 g}$ & 16 & 32 & 16 & 2 \\
\hline Clotrimazole & 1 & 0.5 & 4 & \\
\hline
\end{tabular}

\section{Structure-activity relationship}

The structure-activity relationship of target compound and the standard is shown in Fig. 1. Compound 4e exhibited high larvicidal and nematicidal activities owing to the presence of pyridine with thiopyran moiety besides $\mathrm{CH}_{3} \mathrm{O}$ group. The lower degree of larvicidal and nematicidal activities showed by the compounds $2 \mathbf{a}-\mathrm{g}$ could be due to the presence of piperidine with pyridine rings. 


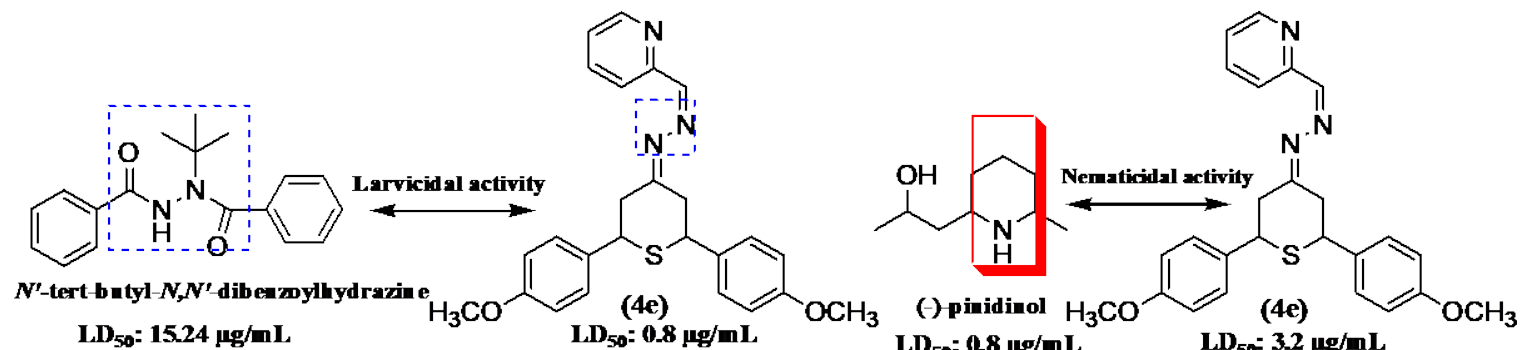

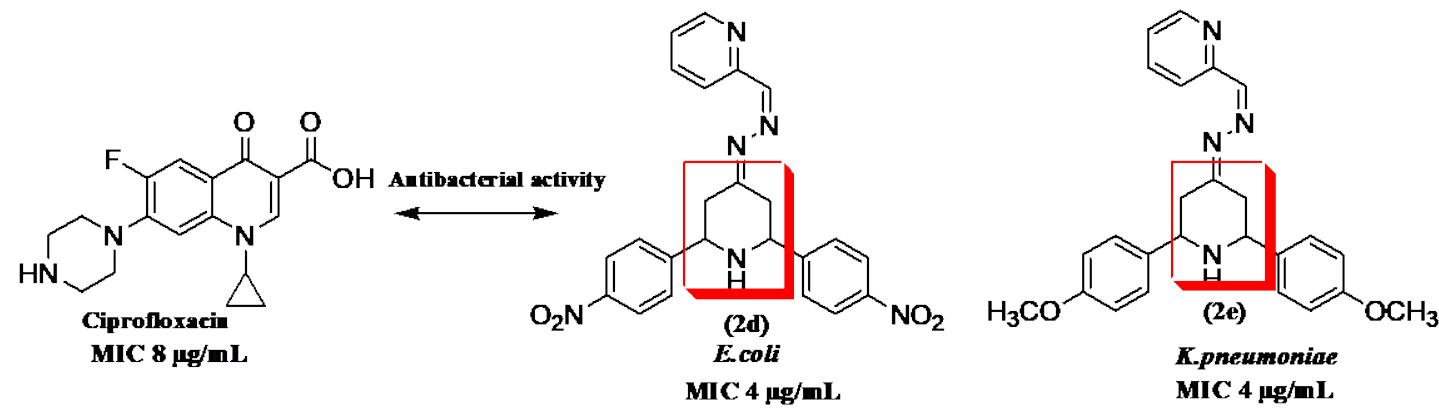<smiles>Clc1ccccc1C(c1ccccc1)(c1ccccc1)n1ccnc1</smiles>

Clotrimazole MIC 0.5 $\mu \mathrm{g} / \mathrm{mL}$

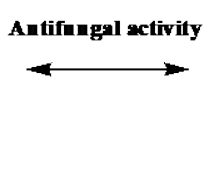
(Cl) Calbicans MIC 0.25 $\mu \mathrm{g} / \mathrm{mL}$

Fig. 1. Structure activity relationship.

The high antibacterial activity of compound $2 \mathbf{e}$ against $K$. pneumoniae is due to presence of pyridine with piperidine moiety and $\mathrm{CH}_{3} \mathrm{O}$ group (MIC: $4 \mu \mathrm{g} / \mathrm{mL}$ ). The presence of pyridine with piperidine moiety and $\mathrm{NO}_{2}$ group is responsible for the high antibacterial activity of compound 2d against E.coli (MIC: 4 $\mu \mathrm{g} / \mathrm{mL}$ ). The 4-substituted phenyl ring acts as a lipophilic domain, and the $\mathrm{NH}$ group present in piperidine act as a hydrogen bonding domain. It can be suggested that piperidine ring is an essential pharmacophore for antibacterial activity.

The presence of thiopyran moiety with C-l-substituted benzene groups was responsible for the high antifungal activity of compound $\mathbf{4 b}$ against $C$. albicans (MIC: $0.25 \mu \mathrm{g} / \mathrm{mL}$ ). Compound $\mathbf{4 f}$ showed high activity against $M$. audouinii due to the presence of thiopyran moiety with methyl substituted benzene groups (MIC: $2 \mu \mathrm{g} / \mathrm{mL}$ ). Notably, the thiopyran moiety showed significant antifungal activities.

In our previous study [22], the compounds (piperidin-connected 2-thioxoimidazolidin-4-one) were found to be lethal to the second instar larvae of mosquito, which produced a $\mathrm{LD}_{50}$ value of $1.37 \mu \mathrm{g} / \mathrm{mL}$ as compared to pinidinol and hyantocidin ( $\mathrm{LD}_{50}$ values of 18.28 and $22.11 \mu \mathrm{g} / \mathrm{mL}$ respectively), however it was low active as compared to compound $\mathbf{4} \mathbf{e}$ of present study. Nematicidal activity of previous study [22] showed that the compound (piperidin-connected 2-thioxoimidazolidin-4-one) with $\mathrm{LD}_{50}$ value of $1.57 \mu \mathrm{g} / \mathrm{mL}$ demonstrated high activity as compared to pinidinol and hyantocidin $\left(\mathrm{LD}_{50}\right.$ values of $14.25,26.30 \mu \mathrm{g} / \mathrm{mL}$ respectively) but very low active in comparison to compound $\mathbf{4 e}$. Therefore, according to the previous study, the 2-thioxoimidazolidin-4-one with piperidin ring showed low potentiality as compared with compound 4e against mosquito larvae and nematodes. At the same time results of the present study evidenced that piperidine series has very low activity as compared to thiopyran $\mathbf{4 a - 4 g}$ series. 


\section{Conclusions}

Compounds 2a-g and 4a-g were synthesized and screened for larvicidal, nematicidal, and antimicrobial activities. Among the synthesized compounds, 4e showed high larvicidal and nematicidal activities. Compounds $\mathbf{2 e}$ and $\mathbf{2 d}$ showed high antibacterial activity against $K$. pneumoniae and E. coli, respectively. Compound $\mathbf{4 b}$ exhibited high antifungal activity against $C$. albicans. Therefore overall results of the present study envisaged that compounds $\mathbf{2 e}, \mathbf{2 d}, \mathbf{4 e}, \mathbf{4 b}$, and $\mathbf{4 f}$ can be used as lead molecules for the development of larvicidal, nematicidal, and antimicrobial agents in future.

\section{Acknowledgements}

The project was supported by King Saud University, Deanship of Scientific Research, Research Chair. We are very grateful to Prince Sultan Research Chair for Environment and Wildlife \& Saudi Biological Society. We thank the Department of Botany \& Microbiology, College of Sciences, King Saud University (KSU), Riyadh, Saudi Arabia for encouragement and support for funding this work.

\section{References}

1. Nagano, R.; Adachi, Y.; Imamura, H.; Yamada, K.; Hashizume, T.; Morishima, H. Antimicrob. Agents Chemother. 1999, 43, 2497-2503.

2. Chang Yong, H.; Young Kwan, K.; Jay Hyok, C.; Se Ho, K.; Hoon, C.; Do Hyun, N. J. Med. Chem. 1997, 40, 3584-3593.

3. Stefania, M.; Maddalena, R.; Piero, V.; Paolo, D. R. Farmaco 1999, 54, 411-415.

4. Kai, L.; Ming-Liang, L.; Lian-Shun, F.; Lan-Ying, S.; Ye-Xin, S.; Zeng-Quan, W. Eur. J. Med. Chem. 2012, 47, 619-625.

5. Park, I. K.; Lee, S. G.; Shin, S. C.; Park, J. D.; Ahin, Y. J. J. Agric.Food. Chem. 2002, 50, 18661870.

6. Perumal, R.V.; Adiraj, M. A.; Shanmugagpandiyan, P. Indian Drugs 2001, 381, 156-159.

7. Hagenbach, R. E.; Gysin, H. Experentia 1952, 8, 184-188.

8. Ilenna, B.; Dobre, V.; Nicluescu-duvaz, I. J. Prakt. Chem. 1985, 327, 667-674.

9. Ramalingan, C.; Balasubramanian Kabilan, S.; Vasudevan, M. Med. Chem. Res. 2003, 12, 41-55.

10. Schneller, S. Adv. Heterocycl. Chem.1975, 18, 59-97.

11. Casy, A. F; Coates, J. E.; Rostron, C. J. Pharm. Pharmacol. 1976, 28, 106-110.

12. Quaglia, W.; Pigini, M.; Piergentili, A.; Giannella, M.; Gentili, F.; Marucci, G.; Carrieri, A.; Carotti, A.; Poggesi, E.; Leonardi, A.; Melchiorre, C. J. Med. Chem. 2002, 45, 1633-1643.

13. Van Vliet, L.A.; Rodenhuis, N.; Dijkstra, D.; Wikström, H.; Pugsley, T. A.; Serpa, K. A.; Meltzer, L. T.; Heffner, T. G.; Wise, L. D.; Lajiness, M. E.; Huff, R. M.; Svensson, K.; Sundell, S.; Lundmark, M. J. Med. Chem. 2000, 43, 2871-2882.

14. Rogier, J. D. J.; Carter, J. S.; Talley, J. J. Patent CA 2001049675A1 2001.

15. Yang, Y. C.; Lee, S. G.; Lee, H. K.; Kim, M. K.; Lee, S. H.; Lee, H. S. J. Agric. Food. Chem . 2002, 50, 3765-37.

16. Sun, R.; Li, Y.; Lue, M.; Xiong, L.; Wang, Q. Bioorg. Med. Chem. Lett. 2010, 20, 4693-4699.

17. Talontsi, F. M.; Matasyoh, J. C.; Ngoumfo, R. M.; Chepkorir, R. Pest. Biochem. Physiol. 2011, 99, 82-85.

18. Wing, K. D. Science 1988, 241, 467-469.

19. Manilal, A.; Thajuddin, N.; Selvin, J.; Idhayadhulla, A. J. Zool. Res. 2011, 7, 272-278.

20. Chitwood, D. J. Pest. Manag. Sci. 2003, 59, 748-753. 
21. Keiser, J.; Utzinger, J. J. Am. Med. Assoc. 2008, 299, 1937-1948.

22. Arif, I. A.; Anis, A.; Surendra Kumar, R.; Idhayadhulla, A.; Aseer, M. Saudi J. Biol. Sci. 2018, in press.

23. Bauer, A. W.; Kirby, W. M.; Sherris, J. C.; Turck, M. Am. J. Clin. Pathol. 1966, 39, 493-496.

24. Idhayadhulla, A.; Surendra Kumar, R.; Jamal Abdul Nasser, A. J. Mex. Chem. Soc, 2011, 55, 218223.

25. Wardlaw, A. C. Practical statistics for experimental biologists, John Wiley and Sons, Chichester (1985)

26. Baliah, V.; Noller, C. R. J. Am. Chem. Soc. 1948, 70, 3853-3858.

27. Sampath, N.; Ponnuswamy, M. N.; Nethaji, M. Cryst. Res. Technol. 2006, 41, 192-197.

28. Petersdorf, R. G.; Sherris, J. C. Am. J. Med. 1965, 39, 766-779. 